\title{
THE CRITICAL TEMPERATURE FOR THE BCS EQUATION AT WEAK COUPLING
}

\author{
RUPERT L. FRANK, CHRISTIAN HAINZL, SERGUEI NABOKO, \\ AND ROBERT SEIRINGER ${ }^{\dagger}$
}

\begin{abstract}
For the BCS equation with local two-body interaction $\lambda V(x)$, we give a rigorous analysis of the asymptotic behavior of the critical temperature as $\lambda \rightarrow 0$. We derive necessary and sufficient conditions on $V(x)$ for the existence of a non-trivial solution for all values of $\lambda>0$.
\end{abstract}

\section{INTRODUCTION}

The BCS model has played a prominent role in condensed matter physics in the fifty years since its introduction 2]. Originally introduced as a model for electrons displaying superconductivity, it has recently also been used to describe dilute cold gases of fermionic atoms in the case of weak interactions among the atoms [10, 12, 14, 1, 13, 4, 3. We will not be concerned here with a mathematical justification of the approximations leading to the BCS model, but rather with an investigation of its precise predictions.

We consider the BCS equation for a Fermi gas at chemical potential $\mu \in \mathbb{R}$ and temperature $T>0$, with local two-body interaction $2 \lambda V(x)$. Here, $\lambda>0$ denotes a coupling constant, and the factor 2 is introduced for convenience. Because of the many different applications of the BCS equation, it is important to keep the discussion as general as possible. Our only assumption on the interaction potential $V$ will be that it is real-valued and $V \in L^{1}\left(\mathbb{R}^{3}\right) \cap L^{3 / 2}\left(\mathbb{R}^{3}\right)$.

It was shown in 8 , that the existence of a non-trivial solution to the BCS gap equation

$$
\Delta(p)=-\frac{\lambda}{(2 \pi)^{3 / 2}} \int_{\mathbb{R}^{3}} \hat{V}(p-q) \frac{\Delta(q)}{E(q)} \tanh \frac{E(q)}{2 T} d q
$$

with $E(p)=\sqrt{\left(p^{2}-\mu\right)^{2}+|\Delta(p)|^{2}}$ at some temperature $T>0$ is equivalent to the fact that a certain linear operator has a negative eigenvalue. Here, $\hat{V}(p)=$ $(2 \pi)^{-3 / 2} \int V(x) e^{-i p x} d x$ denotes the Fourier transform of $V(x)$. In particular, it was shown that this property holds for $T$ less than a certain critical temperature, which we denote by $T_{c}$, whereas there are no non-trivial solutions to Eq. (1) for $T \geq T_{c}$. According to the usual interpretation of solutions to the BCS gap equation, the system displays superfluid behavior for all temperatures $T<T_{c}$, while it is in a normal phase for $T \geq T_{c}$. The analysis in 8 shows that $T_{c}$ is non-zero for purely attractive (i.e., non-positive) $V$, and exponentially small in $\lambda$.

Date: October 22, 2007.

(C) 2007 by the authors. This paper may be reproduced, in its entirety, for non-commercial purposes.

$\dagger$ R.S. acknowledges partial support by U.S. NSF grant PHY-0353181 and by an A.P. Sloan Fellowship. 
The fact that the critical temperature in the non-linear BCS equation can be expressed in terms of spectral properties of a linear operator allows for a more thorough investigation of its properties. This is the purpose of this paper. In particular, we shall be concerned here with the asymptotic behavior of $T_{c}$ at weak coupling, i.e., for small $\lambda$. We shall derive necessary and sufficient conditions on $V$ for the positivity of $T_{c}$ for all $\lambda>0$, as well as its precise asymptotics as $\lambda \rightarrow 0$. The precise statement of our results is given in Theorem 1 below.

The linear operator one is led to analyze is of the form $K_{T, \mu}+\lambda V$ where $K_{T, \mu}$ is a multiplication operator in momentum space that represents an 'effective' kinetic energy. By a modification of the Birman-Schwinger principle we need to study the diverging part of the compact operator

$$
(\operatorname{sgn} V)|V|^{1 / 2} K_{T, \mu}^{-1}|V|^{1 / 2}
$$

as $T \rightarrow 0$. Note that, if $V$ is not of definite $\operatorname{sign}$, then the latter operator is not selfadjoint and standard perturbation arguments based on the variational principle will fail. Still we are able to give a variational characterization for the leading behavior of the critical temperature in the weak coupling limit.

Our analysis is somewhat similar in spirit to that of the lowest eigenvalue of the Schrödinger operator $p^{2}+\lambda V$ in two space dimensions, see [15. This latter case is considerably simpler, however, since $p^{2}$ has a unique minimum at $p=0$, whereas $K_{T, \mu}(p)$ takes its minimal value on the Fermi sphere $p^{2}=\mu$. Technically, this is reflected in the fact that the singular part of the Birman-Schwinger operator $(\operatorname{sgn} V)|V|^{1 / 2}\left(p^{2}+T\right)^{-1}|V|^{1 / 2}$ is of rank one in contrast to that of (2), which is of infinite rank. In particular, the difficulties stemming from the non-selfadjointness are not present in the case of $p^{2}+\lambda V$.

We would like to emphasize that our approach is not restricted to the kinetic energy $K_{T, \mu}$ appearing in the BCS model, but can be adopted to any symbol vanishing on a manifold of codimension one or higher. Operators of this form arise naturally in various fields of Mathematical Physics, e.g. in the quantum-mechanical description of particles in a homogeneous magnetic field or in the analysis of trapped modes in elasticity theory [5, 6, 9, 16].

\section{MAIL RESUlts AND DISCUSSION}

According to the analysis in [8, the critical temperature in the BCS model is, in appropriate units, given by the following expression.

DEFINITION 1. For $\mu \in \mathbb{R}$ and $T>0$, let $K_{T, \mu}$ be the multiplication operator in momentum space

$$
K_{T, \mu}(p)=\left(p^{2}-\mu\right) \frac{e^{\left(p^{2}-\mu\right) / T}+1}{e^{\left(p^{2}-\mu\right) / T}-1} .
$$

Let $V \in L^{1}\left(\mathbb{R}^{3}\right) \cap L^{1}\left(\mathbb{R}^{3}\right)$ be real-valued. The critical temperature in the BCS model is given by

$$
T_{c}(V)=\inf \left\{T>0: \inf \operatorname{spec}\left(K_{T, \mu}+V\right) \geq 0\right\} .
$$

More precisely, it was shown in [8] that Eq. (1) has a non-trivial solution for $T<T_{c}(\lambda V)$, whereas for $T \geq T_{c}(\lambda V)$ it doesn't. Note that $K_{T, \mu} \geq 2 T$, and that the essential spectrum of $K_{T, \mu}+V$ is $[2 T, \infty)$. Hence, in case $T_{c}(V)>0$, it is the 
largest $T$ such that $K_{T, \mu}+V$ has a zero eigenvalue. Note also that $K_{T, \mu}$ becomes $\left|p^{2}-\mu\right|$ as $T \rightarrow 0$.

We assume that $\mu>0$ henceforth. For weak potentials $V$, the critical temperature is determined by the behavior of the potential on the Fermi sphere $\Omega_{\mu}$, the sphere in momentum space with radius $\sqrt{\mu}$. We denote the Lebesgue measure on $\Omega_{\mu}$ by $d \omega$.

Let $\mathcal{V}_{\mu}: L^{2}\left(\Omega_{\mu}\right) \rightarrow L^{2}\left(\Omega_{\mu}\right)$ be the self-adjoint operator

$$
\left(\mathcal{V}_{\mu} u\right)(p)=\frac{1}{(2 \pi)^{3 / 2}} \frac{1}{\sqrt{\mu}} \int_{\Omega_{\mu}} \hat{V}(p-q) u(q) d \omega(q) .
$$

We note that $\mathcal{V}_{\mu}$ is non-vanishing if $\hat{V}(p)$ does not vanish identically for $|p| \leq 2 \sqrt{\mu}$. Since $V \in L^{1}\left(\mathbb{R}^{3}\right)$ by assumption, $\hat{V}$ is a bounded continuous function, and hence $\mathcal{V}_{\mu}$ is a Hilbert-Schmidt operator. It is, in fact, trace class, as will be shown below, and its trace equals $\frac{\sqrt{\mu}}{2 \pi^{2}} \int V(x) d x$.

Let $a_{\mu}(V)=\inf \operatorname{spec}\left(\mathcal{V}_{\mu}\right)$ denote the infimum of the spectrum of $\mathcal{V}_{\mu}$. Since $\mathcal{V}_{\mu}$ is compact, we have $a_{\mu}(V) \leq 0$. Note that, in particular, $a_{\mu}(V)$ is negative if the trace of $\mathcal{V}_{\mu}$ is negative, that is, $a_{\mu}(V)<0$ if $\hat{V}(0)=(2 \pi)^{-3 / 2} \int V(x) d x<0$. Moreover, by considering a trial function that is supported on two small sets on the Fermi sphere separated a distance $|p|$, it is easy to see that $a_{\mu}(V)<0$ if $|\hat{V}(p)|>\hat{V}(0)$ for some $p$ with $|p|<2 \sqrt{\mu}$.

Our main result concerning the critical temperature (3) is as follows.

THEOREM 1. Let $V \in L^{3 / 2}\left(\mathbb{R}^{3}\right) \cap L^{1}\left(\mathbb{R}^{3}\right)$ be real-valued, and let $\lambda>0$.

(i) Assume that $a_{\mu}(V)<0$. Then $T_{c}(\lambda V)$ is non-zero for all $\lambda>0$, and

$$
\lim _{\lambda \rightarrow 0} \lambda \ln \frac{\mu}{T_{c}(\lambda V)}=-\frac{1}{a_{\mu}(V)} .
$$

(ii) Assume that $a_{\mu}(V)=0$. If $T_{c}(\lambda V)$ is non-zero, then $\ln \left(\mu / T_{c}(\lambda V)\right) \geq c \lambda^{-2}$ for some $c>0$ and small $\lambda$.

(iii) If there exists an $\epsilon>0$ such that $a_{\mu}(V-\epsilon|V|)=0$, then $T_{c}(\lambda V)=0$ for small enough $\lambda$.

Note that Eq. (5) implies that, in case $a_{\mu}(V)<0$, the critical temperature has the asymptotic behavior

$$
T_{c}(\lambda V) \sim \mu e^{1 /\left(\lambda a_{\mu}(V)\right)}
$$

in the limit of small $\lambda$. On the other hand, if $a_{\mu}(V)=0$ then part (ii) of Theorem 1 implies that $T_{c}(\lambda V)$ is at most as big as $e^{- \text {const } / \lambda^{2}}$ for some positive constant. If $a_{\mu}(V)$ remains zero if $\epsilon|V(x)|$ is subtracted from $V(x)$, then $T_{c}(\lambda V)=0$ for small enough $\lambda$, and there is no superfluid phase at weak coupling.

Although we restrict our attention to local potentials $V$ here, we remark that a similar analysis can be applied in the case of non-local potentials as well.

2.1. Radial Potentials. In the special case of radial potentials $V$, depending only on $|x|$, the spectrum of $\mathcal{V}_{\mu}$ can be determined more explicitly. Since $\mathcal{V}_{\mu}$ commutes with rotations in this case, all its eigenfunctions are given by spherical harmonics. For $\ell$ a non-negative integer, the eigenvalues of $\mathcal{V}_{\mu}$ are then given by $\frac{\sqrt{\mu}}{2 \pi^{2}} \int V(x)\left|j_{\ell}(\sqrt{\mu}|x|)\right|^{2} d x$, with $j_{\ell}$ denoting the spherical Bessel functions. These 
eigenvalues are $(2 \ell+1)$ fold degenerate. In particular, we then have

$$
a_{\mu}(V)=\inf _{\ell \in \mathbb{N}} \frac{\sqrt{\mu}}{2 \pi^{2}} \int V(x)\left|j_{\ell}(\sqrt{\mu}|x|)\right|^{2} d x .
$$

in the case of radial potentials $V$. We remark that $\sum_{\ell \in \mathbb{N}}(2 \ell+1)\left|j_{\ell}(r)\right|^{2}=1$, hence the expression for the trace of $\mathcal{V}_{\mu}$ stated above is recovered.

If $\hat{V}$ is non-positive, it is easy to see that the infimum is attained at $\ell=0$. This follows since the lowest eigenfunction can be chosen non-negative in this case, and is thus not orthogonal to the constant function. Since $j_{0}(r)=\sin (r) / r$, this means that $a_{\mu}(V)=\left(2 \pi^{2} \sqrt{\mu}\right)^{-1} \int V(x) \frac{\sin ^{2}(\sqrt{\mu}|x|)}{|x|^{2}} d x$ for radial potentials $V$ with non-positive Fourier transform.

In the limit of small $\mu$ we can use the asymptotics $j_{\ell}(r) \approx r^{\ell} /(2 \ell+1) !$ ! to observe that, in case $\int V(x) d x<0, a_{\mu}(V) \approx \frac{\sqrt{\mu}}{2 \pi^{2}} \int V(x) d x$ as $\mu \rightarrow 0$. Note that $(\lambda / 4 \pi) \int V(x) d x$ is the first Born approximation to the scattering length of $2 \lambda V$, which we denote by $a_{0}$. Thus, replacing $\lambda a_{\mu}(V)$ by $2 \sqrt{\mu} a_{0} / \pi$ and writing $\mu=k_{\mathrm{f}}^{2}$, we arrive at the expression $T_{c} \sim e^{\pi /\left(2 k_{\mathrm{f}} a_{0}\right)}$ for the critical temperature, which is well established in the physics literature [7, 12, 3].

In the remainder of this paper, we shall give the proof of Theorem 1

\section{Proof of Theorem 1}

Note that in case $T_{c}>0$, the essential spectrum of $K_{T_{c}, \mu}+\lambda V$ starts at $2 T_{c}>0$, and hence $T_{c}$ is the largest $T$ such that 0 is an eigenvalue of $K_{T, \mu}+\lambda V$ in this case. Therefore there exists an eigenstate $|\psi\rangle \in L^{2}\left(\mathbb{R}^{3}\right)$ such that $K_{T_{c}, \mu}|\psi\rangle=-\lambda V|\psi\rangle$. For a (not necessarily sign-definite) potential $V(x)$ let us use the notation

$$
V(x)^{1 / 2}=(\operatorname{sgn} V(x))|V(x)|^{1 / 2} .
$$

The Birman-Schwinger principle then implies that $|\varphi\rangle=V^{1 / 2}|\psi\rangle$ satisfies $B_{T_{c}}|\varphi\rangle=$ $-|\varphi\rangle$, where

$$
B_{T}=\lambda V^{1 / 2} K_{T, \mu}^{-1}|V|^{1 / 2}
$$

Conversely, if $B_{T}|\varphi\rangle=-|\varphi\rangle$ and $|\psi\rangle=K_{T, \mu}^{-1}|V|^{1 / 2}|\varphi\rangle$, then $|\psi\rangle \in L^{2}\left(\mathbb{R}^{3}\right)$ and $K_{T, \mu}|\psi\rangle=-\lambda V|\psi\rangle$. The existence of a zero eigenvalue for $K_{T, \mu}+\lambda V$ is thus equivalent to the fact that $B_{T}$ has an eigenvalue -1 . Note that $B_{T}$ is not a selfadjoint operator, however.

With the aid of the Birman-Schwinger operator $B_{T}$, we can thus state the following alternative characterization of the critical temperature $T_{c}(\lambda V)$.

Lemma 1. For any $T>0$, the Birman-Schwinger operator $B_{T}$ defined in (6) is Hilbert-Schmidt and has real spectrum. If $T_{c}(\lambda V)>0$, the smallest eigenvalue of $B_{T_{c}}$ equals -1 . Moreover, in case $T_{c}(\lambda V)=0$, the spectrum of $B_{T}$ is contained in $(-1, \infty)$ for any $T>0$.

Proof. The Hilbert-Schmidt property follows from the Hardy-Littlewood-Sobolev inequality [11, Thm. 4.3], using that $V \in L^{3 / 2}\left(\mathbb{R}^{3}\right)$ and that $K_{T, \mu} \geq \operatorname{const}\left(1+p^{2}\right)$. Moreover, $B_{T}$ is the product of a self-adjoint operator (multiplication by $\operatorname{sgn}(V(x))$ ) and a non-negative operator, hence it has real spectrum.

We have already shown above that -1 is an eigenvalue of $B_{T_{c}}$ in case $T_{c}>0$. Moreover, because of strict monotonicity of $K_{T, \mu}$ in $T,-1$ is not an eigenvalue of $B_{T}$ for all $T>T_{c}$. This implies that $B_{T_{c}}$ has no eigenvalue less than -1 , for otherwise 
there would be a $T>T_{c}$ for which $B_{T}$ has eigenvalue -1 since the eigenvalues of $B_{T}$ depend continuously on $T$ and approach 0 as $T \rightarrow \infty$.

In the same way, one argues that $B_{T}$ does not have an eigenvalue less than or equal to -1 if $T_{c}=0$.

Let $J$ be the unitary operator that multiplies by $\operatorname{sgn}(V(x))$. To be precise, we define $\operatorname{sgn}(V(x))=1$ in case $V(x)=0$. Moreover, let $X$ denote the self-adjoint operator on $L^{2}\left(\mathbb{R}^{3}\right)$ with integral kernel

$$
X(x, y)=|V(x)|^{1 / 2} \frac{1}{2 \pi^{2}} \frac{\sin \sqrt{\mu}|x-y|}{|x-y|}|V(y)|^{1 / 2} .
$$

We note that the $X$ is a non-negative trace-class operator, with trace $\operatorname{tr}[X]=$ $\frac{\sqrt{\mu}}{2 \pi^{2}} \int|V(x)| d x$. Hence also $J X$ is trace-class, and $\operatorname{tr}[J X]=\frac{\sqrt{\mu}}{2 \pi^{2}} \int V(x) d x$. Define $Y_{T}$ by

$$
B_{T}=\lambda \ln \left(1+\frac{\mu}{2 T}\right) J X+\lambda Y_{T}
$$

We have

Lemma 2. Let $V \in L^{1}\left(\mathbb{R}^{3}\right) \cap L^{3 / 2}\left(\mathbb{R}^{3}\right)$. Then, for any $T>0$, the operator $Y_{T}$ defined in (7) is Hilbert-Schmidt, and its Hilbert-Schmidt norm is bounded uniformly in $T$, i.e., $\sup _{T>0} \operatorname{tr}\left[Y_{T}^{\dagger} Y_{T}\right]<\infty$.

The proof of this lemma will be given in the next section. Lemma 2 shows that the singular part of the operator $B_{T}$ as $T \rightarrow 0$ is given by $J X$. This observation will enable us to recover the exact asymptotics of $T_{c}(\lambda V)$ as $\lambda \rightarrow 0$.

The operator $J X$ is closely related to $\mathcal{V}_{\mu}$ defined in (4). In fact, the two operators are isospectral.

Lemma 3. The spectrum of $J X$ on $L^{2}\left(\mathbb{R}^{3}\right)$ equals the spectrum of $\mathcal{V}_{\mu}$ on $L^{2}\left(\Omega_{\mu}\right)$.

Proof. Let $A: L^{2}\left(\mathbb{R}^{3}\right) \mapsto L^{2}\left(\Omega_{\mu}\right)$ denote the operator which maps $\psi \in L^{2}\left(\mathbb{R}^{3}\right)$ to the Fourier transform of $|V|^{1 / 2} \psi$, restricted to the sphere $\Omega_{\mu}$. Note that $|V|^{1 / 2} \psi \in$ $L^{1}\left(\mathbb{R}^{3}\right)$ and hence it has a bounded and continuous Fourier transform. Moreover, let $B: L^{2}\left(\Omega_{\mu}\right) \mapsto L^{2}\left(\mathbb{R}^{3}\right)$ be defined by

$$
(B u)(x)=V(x)^{1 / 2} \frac{1}{(2 \pi)^{3 / 2}} \frac{1}{\sqrt{\mu}} \int_{\Omega_{\mu}} u(p) e^{i p x} d \omega(p) .
$$

Using the fact that $\int_{\Omega_{\mu}} e^{i p x} d \omega(p)=4 \pi \sqrt{\mu}|x|^{-1} \sin \sqrt{\mu}|x|$ it is easy to see that $J X=B A$, while $A B=\mathcal{V}_{\mu}$. Hence they have the same spectrum, except possibly at zero. Indeed, if $A B|f\rangle=\lambda|f\rangle$ with $\lambda \neq 0$, then $|g\rangle=B|f\rangle \neq 0$ and $B A|g\rangle=\lambda|g\rangle$. Since both operators are Hilbert-Schmidt operators on infinite-dimensional spaces, 0 is an element of both spectra.

We now study the behavior of the spectrum of $J X$ under small perturbations. We will show that for $\alpha>0$ the spectrum of

$$
\alpha J X+\lambda Y_{T}
$$

differs from the spectrum of $\alpha J X$ by at most $\Theta(\sqrt{\alpha \lambda})+\Theta(\lambda)$, uniformly in $T$. Here and in the following, we use the notation $\Theta(t)$ to indicate an expression that is bounded as $c t \leq \Theta(t) \leq C t$ for constants $0<c<C$. 
Pick a $z$ that stays away a distance $d$ from the spectrum of $\alpha J X$. By expanding in a Neumann series, we see that $\alpha J X+\lambda Y_{T}-z$ has a bounded inverse provided

$$
\lambda\left\|Y_{T}\right\|\left\|\frac{1}{\alpha J X-z}\right\|<1
$$

We have

$$
\frac{1}{\alpha J X-z}=-\frac{1}{z}+\frac{\alpha}{z} J X^{1 / 2} \frac{1}{\alpha X^{1 / 2} J X^{1 / 2}-z} X^{1 / 2} .
$$

Since $X^{1 / 2} J X^{1 / 2}$ is a self-adjoint operator having the same spectrum as $J X$, we can bound $\left\|1 /\left(\alpha X^{1 / 2} J X^{1 / 2}-z\right)\right\| \leq 1 / d$ for any $z$ a distance $d$ away from the spectrum of $\alpha J X$. We conclude that $\left\|(\alpha J X-z)^{-1}\right\| \leq 1 / d+\alpha\|X\| / d^{2}$. Hence $z$ is not in the spectrum of (8) if $d \geq \Theta(\sqrt{\lambda \alpha})+\Theta(\lambda)$.

Since the spectrum of $\alpha J X+\lambda Y_{T}$ depends continuously on $\lambda$, we have thus proved the claim. In particular, it follows that the lowest eigenvalue of (8) equals the lowest eigenvalue of $\alpha J X$ plus terms that are at most of order $\Theta(\sqrt{\alpha \lambda})+\Theta(\lambda)$.

We now have the necessary prerequisites to give the proof of Theorem 1

Proof of Part (i). According to Lemma 3, we have $a_{\mu}(V)=\inf \operatorname{spec} J X$. Assume now that inf spec $J X<0$. Since $Y_{T}$ is bounded uniformly in $T$, we see that the spectrum of $B_{T}=\lambda \ln (1+\mu / 2 T) J X+\lambda Y_{T}$ becomes arbitrarily negative for $T \rightarrow 0$, and hence $T_{c}(\lambda V)>0$ for any $\lambda>0$. Moreover, $\lambda \ln \left(1+\mu / 2 T_{c}\right)$ is bounded away from zero as $\lambda \rightarrow 0$.

We have shown above that the lowest eigenvalue of $B_{T}$ is bounded from above and below by $\lambda a_{\mu}(V) \ln (1+\mu / 2 T)+\Theta(\sqrt{\lambda})$, uniformly in $T$. Since at $T=T_{c}$ this lowest eigenvalues equals -1 , we conclude Eq. (5).

Proof of Part (ii). For $T>0$, let $\alpha=\lambda \ln (1+\mu / 2 T)$ for simplicity. Under the assumption that the spectrum of $J X$ is non-negative, the lowest eigenvalue of $B_{T}=$ $\alpha J X+\lambda Y_{T}$ is bigger than $-\Theta(\sqrt{\alpha \lambda})$, as shown above. This immediately implies that $B_{T}$ can only have an eigenvalue -1 if $\alpha \lambda \geq \Theta(1)$, or $\ln (\mu / T) \geq \Theta\left(1 / \lambda^{2}\right)$ for small $\lambda$.

Proof of Part (iii). Let again $\alpha=\lambda \ln (1+\mu / 2 T)$, and recall that $B_{T}=\alpha J X+\lambda Y_{T}$. Since the operator $1+\lambda Y_{T}$ is invertible for small enough $\lambda$, we are able to rewrite

$$
1+B_{T}=\left(1+\lambda Y_{T}\right)\left(1+\left(1+\lambda Y_{T}\right)^{-1} \alpha J X\right) .
$$

Hence $1+B_{T}$ does not have a zero eigenvalue for any $\alpha \geq 0$ if the spectrum of $\left(1+\lambda Y_{T}\right)^{-1} J X$ is non-negative. Note that $J Y_{T}$ is self-adjoint, since $J B_{T}$ is selfadjoint and $J^{2}=1$. Hence $\left(1+\lambda Y_{T}\right)^{-1} J X$ has the same spectrum as the self-adjoint operator

$$
X^{1 / 2} \frac{1}{J+\lambda J Y_{T}} X^{1 / 2}
$$

This operator is non-negative for small $\lambda$ if $X^{1 / 2} J X^{1 / 2} \geq \epsilon X$ for some $\epsilon>0$, since then

$$
\text { (9) }=X^{1 / 2} J X^{1 / 2}-\lambda X^{1 / 2} Y_{T} \frac{1}{J+\lambda J Y_{T}} X^{1 / 2} \geq X\left(\epsilon-\lambda\left\|Y_{T}\right\|\left\|\left(1+\lambda Y_{T}\right)^{-1}\right\|\right) \text {. }
$$

Note that the range of $X$ is dense in the range of $X^{1 / 2}$, and hence it is enough to check the inequality $J \geq \epsilon$ on the range of $X$. Let $|\psi\rangle$ be in the range of $X$, 
i.e., $|\psi\rangle=X|\phi\rangle$ for some $|\phi\rangle \in L^{2}\left(\mathbb{R}^{3}\right)$. Then $\langle\psi \mid J \psi\rangle \geq \epsilon\langle\psi \mid \psi\rangle$ is equivalent to the statement that, for $|\chi\rangle=|V|^{1 / 2}|\phi\rangle$,

$$
\begin{aligned}
& \int_{\Omega_{\mu} \times \Omega_{\mu}} \overline{\hat{\chi}(p)} \hat{V}(p-q) \hat{\chi}(q) d \omega(p) d \omega(q) \\
& \geq \epsilon \int_{\Omega_{\mu} \times \Omega_{\mu}} \overline{\hat{\chi}(p)}|\widehat{V}|(p-q) \hat{\chi}(q) d \omega(p) d \omega(q) .
\end{aligned}
$$

This, in turn, is equivalent to $a_{\mu}(V-\epsilon|V|)=0$. Under this assumption, we have thus shown that, for small enough $\lambda$, the operator $B_{T}$ does not have an eigenvalue -1 , for arbitrary $T>0$. Together with Lemma 1, this proves the claim.

\section{Proof of Lemma 2}

By scaling we may assume that $\mu=1$, and we set $K_{T, 1}=K_{T}$ for simplicity. The operator $K_{T}$ can be rewritten as

$$
K_{T}(p)=\left(\left|p^{2}-1\right|+2 T\right) g\left(\left|p^{2}-1\right| / T\right)
$$

where $g(t)=t\left(1+e^{-t}\right) /\left((t+2)\left(1-e^{-t}\right)\right)$. The integral kernel of $K_{T}^{-1}$ is given by

$$
K_{T}^{-1}(x, y)=\frac{1}{(2 \pi)^{3}} \int_{\mathbb{R}^{3}} \frac{e^{i p(x-y)}}{\left(\left|p^{2}-1\right|+2 T\right) g\left(\left|p^{2}-1\right| / T\right)} d p .
$$

We decompose $K_{T}(p)$ as $K_{T}(p)^{-1}=L_{T}^{(1)}(p)+M_{T}^{(1)}(p)$, where $L_{T}^{(1)}(p)=\theta(\sqrt{2}-$ $|p|) K_{T}(p)^{-1}$ and $M_{T}^{(1)}(p)=\theta(|p|-\sqrt{2}) K_{T}(p)^{-1}$. Since $b=\inf _{t} g(t)>0$ one has

$$
M_{T}^{(1)}(p) \leq \theta(|p|-\sqrt{2}) b^{-1}\left|p^{2}-1\right|^{-1} .
$$

Using that $V \in L^{3 / 2}\left(\mathbb{R}^{3}\right)$, we find with the aid of the Hardy-Littlewood-Sobolev inequality [11, Thm. 4.3] that $\left\|V^{1 / 2} M_{T}^{(1)}|V|^{1 / 2}\right\|_{2}$ is bounded independently of $T$. Here, $\|\cdot\|_{2}=\left(\operatorname{tr}|\cdot|^{2}\right)^{1 / 2}$ denotes the Hilbert-Schmidt norm.

Note that the integral kernel of $L_{T}^{(1)}$ is given by

$$
\frac{1}{2 \pi^{2}} \int_{0}^{\sqrt{2}} \frac{k}{\left(\left|k^{2}-1\right|+2 T\right) g\left(\left|k^{2}-1\right| / T\right)} \frac{\sin k|x-y|}{|x-y|} d k .
$$

We further decompose $L_{T}^{(1)}=L_{T}^{(2)}+M_{T}^{(2)}$, where

$$
L_{T}^{(2)}(x, y)=\frac{1}{2 \pi^{2}} \int_{0}^{\sqrt{2}} \frac{k}{\left(\left|k^{2}-1\right|+2 T\right)} \frac{\sin k|x-y|}{|x-y|} d k .
$$

Estimating $|\sin k| x-y|| \leq \sqrt{2}|x-y|$ for $k \leq \sqrt{2}$ and changing variables one easily finds

$$
\left|M_{T}^{(2)}(x, y)\right| \leq \frac{\sqrt{2}}{2 \pi^{2}} \int_{0}^{1 / T} \frac{1}{t+2}\left(\frac{1}{g(t)}-1\right) d t .
$$

This is bounded independently of $T$ since $1 / g(t)-1 \sim 2 / t$ as $t \rightarrow \infty$. Since $V \in L^{1}\left(\mathbb{R}^{3}\right)$, we can bound $\left\|V^{1 / 2} M_{T}^{(2)}|V|^{1 / 2}\right\|_{2} \leq \int|V(x)| d x \sup _{x, y}\left|M_{T}^{(2)}(x, y)\right|$, and hence we see that also $\left\|V^{1 / 2} M_{T}^{(2)}|V|^{1 / 2}\right\|_{2}$ is bounded uniformly in $T$. 
Finally, we decompose $L_{T}^{(2)}=L_{T}^{(3)}+M_{T}^{(3)}$, where

$$
\begin{aligned}
L_{T}^{(3)}(x, y) & =\frac{1}{2 \pi^{2}} \int_{0}^{\sqrt{2}} \frac{k}{\left(\left|k^{2}-1\right|+2 T\right)} d k \frac{\sin |x-y|}{|x-y|} \\
& =\ln (1 /(2 T)+1) \frac{1}{2 \pi^{2}} \frac{\sin |x-y|}{|x-y|} .
\end{aligned}
$$

Since $|\sin a-\sin b| \leq|a-b|$ one easily sees that

$$
\left|M_{T}^{(3)}(x, y)\right| \leq \frac{1}{2 \pi^{2}} \int_{0}^{\sqrt{2}} \frac{k}{k+1} d k
$$

Again, since $V \in L^{1}\left(\mathbb{R}^{3}\right),\left\|V^{1 / 2} M_{T}^{(3)}|V|^{1 / 2}\right\|_{2}$ is uniformly bounded. This completes the proof.

\section{REFERENCES}

[1] N. Andrenacci, A. Perali, P. Pieri, G.C. Strinati, Density-induced BCS to Bose-Einstein crossover, Phys. Rev. B 60, 12410 (1999)

[2] J. Bardeen, L. Cooper, J. Schrieffer, Theory of Superconductivity, Phys. Rev. 108, 1175-1204 (1957)

[3] I. Bloch, J. Dalibard, W. Zwerger, Many-Body Physics with Ultracold Gases, Preprint arXiv:0704.3011

[4] Q. Chen, J. Stajic, S. Tan, K. Levin, BCS-BEC crossover: From high temperature superconductors to ultracold superfluids, Phys. Rep. 412, 1-88 (2005)

[5] C. Förster, Trapped modes for the elastic plate with a perturbation of Young's modulus, Preprint arXiv:math-ph/0609032

[6] C. Förster, T. Weidl, Trapped modes for an elastic strip with perturbation of the material properties, Quart. J. Mech. Appl. Math. 59, 399-418 (2006)

[7] L.P. Gor'kov, T.K. Melik-Barkhudarov, Contributions to the theory of superfluidity in an imperfect Fermi gas, Soviet Physics JETP 13, 1018 (1961)

[8] C. Hainzl, E. Hamza, R. Seiringer, J.P. Solovej, The BCS model for general pair interactions, Preprint arXiv:math-ph/0703086

[9] A. Laptev, O. Safronov, T. Weidl, Bound State Asymptotics for Elliptic Operators with Strongly Degenerated Symbols, in: Nonlinear problems in mathematical physics and related topics I, pp. 233-246, Int. Math. Ser. (N.Y.), Kluwer/Plenum, New York (2002)

[10] A.J. Leggett, Diatomic Molecules and Cooper Pairs, in Modern trends in the theory of condensed matter, A. Pekalski, R. Przystawa, eds., Springer (1980)

[11] E. Lieb, M. Loss, Analysis, American Mathematical Society (2001)

[12] P. Nozières, S. Schmitt-Rink, Bose Condensation in an Attractive Fermion Gas: From Weak to Strong Coupling Superconductivity, J. Low Temp. Phys. 59, 195-211 (1985)

[13] M. Parish, B. Mihaila, E. Timmermans, K. Blagoev, P. Littlewood, BCS-BEC crossover with a finite-range interaction, Phys. Rev. B 71, 0645131-0645136 (2005)

[14] M. Randeria, in Bose-Einstein Condensation, A. Griffin, D.W. Snoke, S. Stringari, eds., Cambridge University Press (1995)

[15] B. Simon, The bound state of weakly coupled Schrödinger operators in one and two dimensions, Ann. Phys. 97, 279-288 (1976)

[16] A.V. Sobolev, Asymptotic behavior of energy levels of a quantum particle in a homogeneous magnetic field perturbed by an attenuating electric field. I, Probl. Mat. Anal. 9, 67-84 (1984); II, Probl. Mat. Fiz. 11, 232-248 (1986) 
Rupert L. Frank, Department of Mathematics, Royal Institute of Technology, 100 44 Stockholm, Sweden. Current address: Department of Mathematics, Princeton UniverSity, PRInCETON NJ 08544, USA

E-mail address: rlfrank@math.princeton.edu

Christian Hainzl, Departments of Mathematics and Physics, UAB, 1300 University Blvd, Birmingham AL 35294, USA

E-mail address: hainzl@math.uab.edu

Serguei Naboko, Department of Mathematics, UAB, 1300 University Blvd, Birmingham, AL 35294, USA. Permanent address: Department of Mathematical Physics, St. Petersburg University, St. Petersburg, Russia

E-mail address: naboko@math.uab.edu

Robert Seiringer, Department of Physics, Princeton University, Princeton NJ 085420708, USA

E-mail address: rseiring@math.princeton.edu 